\title{
IMPLICANCIAS DEL USO ESTRATÉGICO DE LAS FunCIONES DE LA EVALUACIÓN EN EL LOGRO DE LAS COMPETENCIAS ESPECÍFICAS
}

\author{
IMPLICATIONS OF THE STRATEGIC USE OF EVALUATION FUNCTIONS \\ IN THE ACHIEVEMENT OF SPECIFIC COMPETENCES
}

\author{
Mónica Fabiola Ramos Neyra ${ }^{1}$
}

\section{RESUMEN}

El desarrollo de competencias genéricas y específicas en los futuros profesionales se logra a través de la articulación entre los modelos educativos y curriculares que brindan un sello institucional al perfil de egreso de los estudiantes según sea la carrera profesional abordada, la sistematización de prácticas docentes que evidencien un uso estratégico de la metodología y didáctica así como de la evaluación. En este artículo, se abordan las implicancias entre el uso estratégico de la función diagnóstica, formativa y sumativa de la evaluación frente a los niveles de logro de las competencias específicas.

Palabras claves: Evaluación, competencias, funciones, evaluación diagnóstica, evaluación formativa, evaluación sumativa, competencias.

\begin{abstract}
The development of generic and specific skills in future professionals is achieved through the articulation between educational and curricular models that provide an institutional sign to the student's graduation profile, according to the addressed professional career and the systematization of teaching practices that evidences a strategic use of the methodology and didactic as well as the evaluation. In this article, the implications between the strategic use of the diagnostic, formative and summative function of the evaluation against the achievement levels of the specific competences are addressed.
\end{abstract}

Key words: evaluation, proficiency, functions, diagnostic evaluation, formative evaluation, summative evaluation, competences.

\section{INTRODUCCIÓN}

$\mathfrak{E}$ n la actualidad, las universidades pertenecen a un macrosistema que evalúa la calidad educativa en función a diferentes criterios que se orientan al mercado laboral, opiniones sobre calidad y prestigio del programa y apoyo académico, entre otros. (1) Estas dimensiones e indicadores evalúan, de manera integral, las políticas y acciones para asegurar la vinculación entre las necesidades del escenario laboral y procesos educativos formativos destinados a lograr el desarrollo de las competencias. $(2,3)$ Las políticas y disposiciones acerca de la evaluación generan un marco que permite que se administre de una forma planificada y metódica, de modo que permita develar el nivel de desempeño de la competencia que se pretende perfeccionar y desarrollar.

1 Profesora del Departamento de Humanidades. Universidad Privada del Norte. 
La Evaluación de los Aprendizajes en EL Aula desde una Perspectiva Competencial $Y$ EsTRATÉGICA

La evaluación, como práctica en el aula, es un constructo que ha variado conceptualmente en la cultura. Desde las prácticas educativas no formalizadas y reconocidas como procesos instruccionales hasta la formación de sistemas educativos en la edad moderna y contemporánea se ha empleado la evaluación como herramienta para certificar los aprendizajes obtenidos. La perspectiva de la evaluación nomotética y relacionada con los enfoque positivista y neopositivista despersonalizó la interacción entre el docente y los estudiantes, en la gran mayoría de los casos.

Los expertos en el movimiento de evaluación formativa y compartida coinciden en la escasa funcionalidad de este enfoque puesto que la estructura, parámetros, criterios de evaluación son establecidos por el docente, sin que necesariamente los componentes de la evaluación hayan sido validados por la comunidad educativa. Dentro de este ejercicio, la evaluación se empleaba con el único fin de certificar qué estudiante aprobaba o reprobaba. Además, se mostraban únicamente los resultados y calificaciones sin considerar qué aspectos diagnosticados podrían servir para mejorar y establecer medidas remediales o proactivas para mejorar la calidad de los aprendizajes. Este contexto formativo presenta pocas probabilidades para la asimilación y afianzamiento de las competencias. (4-7)

Por otro lado, la evaluación educativa en función al enfoque competencial apela no a un fin sino a un proceso sistémico de análisis, reflexión y retroalimentación que permita promover aprendizajes y competencias en función a una diversidad concertada de indicadores, herramientas, métodos, técnicas y agentes de evaluación. $(8,9)$ Respecto al proceso, la mayoría de especialistas en evaluación coincide en su apreciación respecto a que la evaluación de los aprendizajes se basa en un procedimiento que debe ser guiado por prácticas intencionales sistémicas que permitirán perfeccionar los estados de desarrollo de competencias. (10-13)

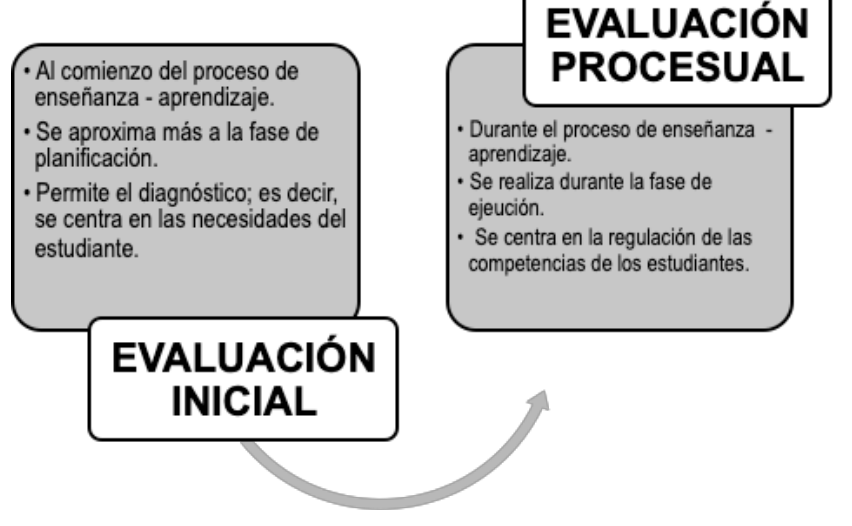

Figura 1: Proceso de evaluación.

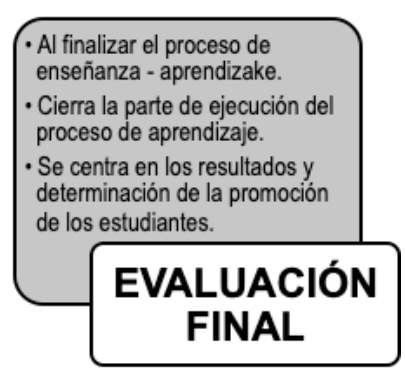

FUENTE: ELABORACIÓN PROPIA 
En la siguiente tabla se puede apreciar las características básicas de las etapas de la evaluación, desde la perspectiva del proceso educativo:

\section{Uso Estratégico de las Funciones De La EVALUACIÓN}

Diversos autores establecen una serie de funciones o atributos propios de la intencionalidad que subyace frente al uso. Como menciona González, si la evaluación es correctamente planificada constituye una fortaleza dentro del proceso educativo. (14) Por un lado, Cardona, citado por Castillo Arredondo \& Cabrerizo Diago, establece cinco funciones básicas de la evaluación ateniéndose únicamente al propósito y no al contexto y momentos en los cuales se produce la evaluación. Para este autor, las funciones son diagnóstica, reguladora, previsora, retro alimentadora y de control. (13) Por otro lado, Posne et al, citados por Mora Vargas explican que las funciones principales de la evaluación son de diagnóstico, instructiva, educativa y autoformadora. (15).

Existe, desde luego, un consenso entre las líneas argumentales de los especialistas respecto al los diversos usos de la evaluación como herramienta de mejora a partir de la detección de necesidades de aprendizaje, falencias en las habilidades o conocimientos básicos y los conocimientos logrados antes de iniciar el proceso de evaluación.

Tabla 1: Características de la evaluación diagnóstica según diversos autores

\begin{tabular}{|c|c|c|}
\hline Gargallo Castel, 2009 (16) & Gonzáles Loayza, 2016 (14) & Castillo \& Cabrerizo, 2010 (13) \\
\hline $\begin{array}{l}\text { Reporte de conocimientos previos. } \\
\text { Ajuste en los contenidos y criterios } \\
\text { de acuerdo con las características del } \\
\text { grupo. } \\
\text { Empleo de distintos formatos de } \\
\text { evaluación. }\end{array}$ & $\begin{array}{l}\text { Adecuación del punto de partida del } \\
\text { proceso de enseñanza - aprendizaje. } \\
\text { Conocimiento las características de los } \\
\text { estudiantes. }\end{array}$ & $\begin{array}{l}\text { Inicio del proceso educativo } \\
\text { con un conocimiento real de las } \\
\text { características de los estudiantes. } \\
\text { Adecuación de las estrategias de } \\
\text { enseñanza y aprendizaje. } \\
\text { Pronóstico de futuros desempeños. }\end{array}$ \\
\hline Ruiz Torres, 2014 (17) & Cifuentes Medina, 2014 (18) & Pimienta \& Tobón (8) \\
\hline $\begin{array}{l}\text { Inicial: identifica los conocimientos } \\
\text { prerrequisito. } \\
\text { Puntual: identifica los conocimientos } \\
\text { previos en cualquier momento del } \\
\text { curso educativo. }\end{array}$ & $\begin{array}{l}\text { Corresponde a la fase de planificación. } \\
\text { Permite tomar las decisiones para } \\
\text { afrontar el hecho educativo. }\end{array}$ & $\begin{array}{l}\text { Valoración de los aprendizajes } \\
\text { previos en función a las } \\
\text { competencias. } \\
\text { Articulación de estrategias } \\
\text { didácticas y actividades de } \\
\text { aprendizaje. } \\
\text { Determinación de fortalezas, } \\
\text { debilidades y expectativas del } \\
\text { proceso de enseñanza y aprendizaje. }\end{array}$ \\
\hline
\end{tabular}

Nota: Elaboración propia a partir de los conceptos de los autores mencionados. 
En el presente artículo, se abordará las funciones de la evaluación en conjunto con el proceso de evaluación. Para ello, se tomará en cuenta la teoría sistematizada por Casstillo et al, la cual reseña los aportes de Casanova y Stufllebeam.

\section{Evaluación Diagnóstica}

Esta función está asociada a situaciones iniciales de aprendizaje. La finalidad de esta evaluación reside en la recolección de información para determinar el nivel de desarrollo que tienen frente a la(s) competencia(s) que se busca desarrollar en el curso. (14,16-18).

A continuación, se reseñarán las características conceptuales que se derivan de los autores mencionados: ver tabla 1

\section{Evaluación Formativa}

Es la que permite valorar el grado de adquisición de las competencias durante el

Tabla 2: Características de la evaluación formativa según diversos autores

\begin{tabular}{|c|c|c|c|}
\hline Gargallo Castel, 2009 (16) & González Loayza, 2016 (14) & Hamodi, 2015 (19) & Ruiz Torres, 2014(17) \\
\hline \multirow{2}{*}{$\begin{array}{l}\text { Evaluación de la efectividad } \\
\text { de los docentes. } \\
\text { Empleo de técnicas formales o } \\
\text { informales }\end{array}$} & Pimienta \& Tobón, 2010 (8) & Castillo \& Cabrerizo, 2010 (13) & \\
\hline & $\begin{array}{l}\text { Retroalimentación sobre } \\
\text { el desarrollo de las } \\
\text { competencias. } \\
\text { Aspectos de mejora. } \\
\text { Progreso de los estudiantes. } \\
\text { Ajustes en la mediación } \\
\text { pedagógica. }\end{array}$ & $\begin{array}{l}\text { Acomodación de la práctica } \\
\text { docente. } \\
\text { Información de todos los } \\
\text { elementos del proceso } \\
\text { educativo. } \\
\text { Reorientación de los } \\
\text { aprendizajes. }\end{array}$ & \\
\hline
\end{tabular}

Nota: Elaboración propia a partir de los conceptos de los autores mencionados. 
Tabla 3: Características de la evaluación sumativa según diversos autores

\begin{tabular}{|c|c|c|c|}
\hline Gargallo Castel, 2009 (16) & Gonzáles Loayza, 2016 (14) & Hamodi, 2015 (19) & Ruiz Torres, 214 (17) \\
\hline $\begin{array}{l}\text { Evaluación centrada el } \\
\text { producto. } \\
\text { Comprobación de la } \\
\text { eficacia del curso. } \\
\text { Información sobre } \\
\text { resultados al término de } \\
\text { una fase de aprendizaje } \\
\text { Establece promoción o } \\
\text { repitencia. } \\
\text { Sirve para acreditar el } \\
\text { nivel alcanzado en una } \\
\text { fase de aprendizaje. }\end{array}$ & $\begin{array}{l}\text { Verificación del logro de los } \\
\text { objetivos y competencias } \\
\text { establecidos. } \\
\text { Determinación de la } \\
\text { posición del estudiante en } \\
\text { el grupo }\end{array}$ & $\begin{array}{l}\text { Constatación institucional } \\
\text { acerca del logro de los objetivos. } \\
\text { Ubicación de a los estudiantes } \\
\text { en puestos } \\
\text { Exclusión a quienes no } \\
\text { alcanzaron los objetivos } \\
\text { Comparación de resultados } \\
\text { entre estudiantes y docentes } \\
\text { Establece obligaciones legales a } \\
\text { los profesores }\end{array}$ & $\begin{array}{l}\text { Determinación del } \\
\text { cumplimiento de los } \\
\text { aprendizajes según las } \\
\text { condiciones y criterios } \\
\text { establecidos. } \\
\text { Conclusiones sobre el } \\
\text { grado de eficacia de la } \\
\text { experiencia educativa } \\
\text { Calificativa o } \\
\text { certificativa. }\end{array}$ \\
\hline \multirow{2}{*}{$\begin{array}{l}\text { Establece promoción o } \\
\text { repitencia. } \\
\text { Sirve para acreditar el } \\
\text { nivel alcanzado en una } \\
\text { fase de aprendizaje. }\end{array}$} & Pimienta \& Tobón, 2010 (8) & Castillo \& Cabrerizo, 2010 (13) & \\
\hline & $\begin{array}{l}\text { Grado de desarrollo de la } \\
\text { competencia } \\
\text { Promoción a otros cursos } \\
\text { de la malla curricular. } \\
\text { Determinación de } \\
\text { las competencias } \\
\text { profesionales. }\end{array}$ & $\begin{array}{l}\text { Grado de aprovechamiento del } \\
\text { estudiante en un periodo. } \\
\text { Grado de consecución de } \\
\text { competencias. } \\
\text { Aprobación o desaprobación en } \\
\text { una asignatura. }\end{array}$ & \\
\hline
\end{tabular}

Nota: Elaboración propia a partir de los conceptos de los autores mencionados.

proceso de aprendizaje. Asimismo, sirve para brindar retroalimentación y diseñar estrategias para la mejora del proceso de enseñanza aprendizaje $(6,8,13,19)$.

Al igual que con la evaluación diagnóstica, se ha realizado la revisión de los aportes de diferentes autores respecto a esta función esencial. ver tabla 2

\section{Evaluación Sumativa}

Al finalizar el periodo de aprendizaje se debe contar con una ponderación que certifique en qué grado se cumplieron los objetivos instruccionales o el grado de adquisición de la competencia; asimismo, certificar socialmente estos logros. Este es el sentido y funcionalidad de la evaluación sumatoria. $(8,19)$.

En la tabla 3, se muestran los atributos conceptuales asociados a este proceso:

\section{REFERENCIAS BibLIOGRÁFICAS}

1. Martínez Rizo F. Los rankings de universidades: una visión crítica. Revista de la Educación Superior. 2011;40(157):77-97.

2. CINTERFOR. Formación basada en competencia laboral: situación actual y perspectivas. Montevideo: Cinterfor; 1997.342 p. 
3. Beneitone P, Cesar E, Gonzalez J, Maleta M, Siufi R, Wagenaar G. Reflexiones y perspectivas de la educación superior en América Latina [Internet]. Informe FinalProyecto Tuning-América Latina. Bilbao: Universidad de Deusto; 2004. 1-432 p.

4. Champin D. Evaluación por competencias en la educación médica. Rev Peru Med Exp Salud Publica. 2014;3.

5. Picos AP, López-Pastor VM. Haz lo que yo digo, pero no lo que yo hago: Sistemas de evaluación del alumnado en la formación inicial del profesorado. Rev Educ. 2013;(361):279-305.

6. Hamodi Galán C. La evaluación formativa y compartida en educación superior: Un estudio de caso. Tesis doctoral. Universidad de Valladolid; 2014.

7. García A, Aguilera M, Pérez M, Muñoz G. Evaluación de los aprendizajes en el aula. Opiniones y prácticas de docentes de primaria en México. Instituto Nacional para la Evaluación de la Educación. México D.F.: Instituto Nacional para la Evaluación de la Educación; 2011. 148 p.

8. Pimienta Prieto J, Tobón S. Secuencias didácticas: aprendizaje y evaluación de competencias. Primera. México D.F.: Pearson Education; 2010. 216 p.

9. Tobón S. Formación Basada en Competencias. Pensamiento complejo, diseño curricular y didáctica [Internet]. Bogotá: Ecoe Ediciones; 2013. 310 p.

10. Delgado García AM, Oliver Cuello R. Interacción entre la evaluación continua y la autoevaluación formativa: La potenciación del aprendizaje autónomo. Rev Docencia Univ [Internet]. 2009;(4):1-13.

11. Ortega Paredes M. Evaluación formativa aplicada por los docentes del área de ciencia, tecnología y ambiente en el distrito de Hunter en Arequipa. Universidad Cayetano Heredia; 2015.

12. Cano E. Buenas prácticas en la evaluación de competencias Cinco casos de educación superior. En: Laertes Educación, editor. Laertes Educación; 2014.
13. Castillo Arredondo S, Cabrerizo Diago J. Evaluación educativa de aprendizajes y competencias. Madrid: Pearson Education; 2010. 487 p.

14. Gonzales Loayza C del R. Planificación de la evaluación del aprendizaje en los cursos generales de una facultad de educación de una universidad de Lima. Pontificia Universidad Católica del Perú; 2016.

15. Mora Vargas AI. La evaluación educativa: concepto, períodos y modelos. Actual Investig en Educ.. 2004;4(2):129.

16. Gargallo Castel A. Aproximación al estudio de la evaluación en la universidad: un análisis exploratorio con alumnos semipresenciales. Context Educ. 2009; 12:77-93.

17. Ruiz Torres MZ. Estudio de las competencias trasversales en un modelo de enseñanza y evaluación formativa en la universidad. Universidad de Málaga; 2014.

18. Cifuentes Medina JE. Las prácticas evaluativas: una reflexión pertinente en la modalidad a distancia. Rastros Rostros. 2014;16(30):19-33.

19. Hamodi C, López V, López A. Medios, técnicas e instrumentos de evaluación formativa y compartida del aprendizaje en educación superior. Perfiles Educ. 2015;37(147):146-61.

20. Hamodi C, López Pastor VM, López Pastor AT. Medios, técnicas e instrumentos de evaluación formativa y compartida del aprendizaje en educación superior. Perfiles Educ. 2015;37(147):146-61.

21. Tobón S. Competencias, calidad y educación superior. Bogotá: Editorial Magisterio; 2006. 256 p.

22. Vargas Leyva MR. Diseño curricular por competencias. México D.F.: Asociación Nacional de Facultades y Escuelas de Ingeniería; 2008. 88 p.

23. CINDA. Diseño curricular basado en competencias y aseguramiento de la calidad en la educación superior. Santiago de Chile: CINDA, 2009.

\section{CORRESPONDENCIA:}

monica.ramos@upn.edu.pe 